\title{
Ionization and pair creation in relativistic heavy-ion collisions
}

\author{
K. Rumrich, G. Soff, and W. Greiner \\ Gesellschaft für Schwerionenforschung (GSI), Planckstrasse 1, Postfach 110 552, D-6100 Darmstadt, Germany \\ and Institut für Theoretische Physik der Johann Wolfgang Goethe Universität, Robert-Mayer-Strasse 8-10, Postfach 111932, \\ D-6000 Frankfurt am Main, Germany \\ (Received 9 March 1992)
}

\begin{abstract}
Ionization, pair creation, and electron excitations in relativistic heavy-ion collisions are investigated in the framework of the coupled-channel formalism. Collisions between heavy projectiles and $\mathrm{Pb}^{82+}$ are considered for various bombarding energies in the region $E=500$ up to $2000 \mathrm{MeV} / \mathrm{u}$. Useful symmetry relations for the matrix elements are derived and the influence of gauge transformations onto the coupled-channel equations is explored.
\end{abstract}

PACS number(s): 34.90. $+\mathrm{q}$, 36.10.Dr, 12.20. $-\mathrm{m}, 25.70 .-\mathrm{z}$

\section{INTRODUCTION}

In recent years a new generation of particle accelerator has been constructed. Heavy ions can be accelerated to relativistic energies and for the near future a further increase in performance is expected. Relativistic heavy-ion collisions provide a tool for the investigation of electrons in extremely strong electromagnetic fields. The comparison of theoretical and experimental results allows for a test of quantum electrodynamics under these extreme conditions.

In this paper atomic physics effects in relativistic heavy-ion collisions are studied [1]. Predominantly we consider collisions with finite impact parameter in which the nuclei do not touch. In Fig. 1 the spectrum of the Dirac equation is depicted. Above the boundary $E=+m c^{2}$ and below the boundary $E=-m c^{2}$ the continua are located. In the gap in between, some bound states are indicated. In the hole picture it is assumed that in the ground state all the states of the negative-energy continuum are occupied by electrons. Removing such an electron implies that the remaining hole is interpreted as a positron.

Some possible excitations are depicted schematically in Fig. 1: (1) excitation of an electron from one bound state into another bound state with lower binding energy; (2) ionization of an originally bound electron; (3) excitation of an electron from the negative-energy continuum into a

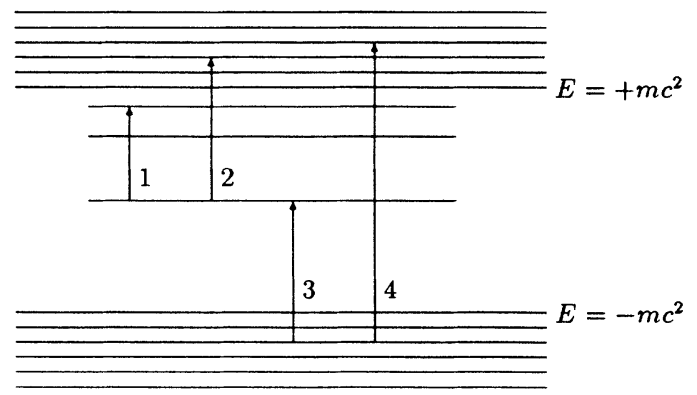

FIG. 1. Spectrum of the Dirac equation. Indicated are possible atomic excitations: (1) excitation, (2) ionization, (3) pair creation with capture, and (4) direct pair creation. bound state. The remaining hole is interpreted as a positron. The created electron is captured in the bound state (pair production with capture). (4) Excitation of an electron from the negative-energy continuum into the positive-energy continuum. As in case (3) this is the creation of an electron-positron pair, but the final state of the electron is in the continuum (direct pair creation).

One additional process is missing in this figure, namely, the capture of an electron initially bound to the target by the impinging projectile. In this paper we will concentrate on excitation, ionization, and pair creation with capture.

Similar calculations are performed by various other authors $[2-6]$. The main differences consist in the chosen basis set and in the methods employed to determine the matrix elements. Former calculations also employed perturbation theory in different forms [6-13]. For collision systems with low- $Z$ projectiles these calculations yield reliable results when compared with experimental data.

A series of experiments has been published by Meyerhof, Anholt, and co-workers. They investigated ionization cross sections $[7,14,15]$, charge states of the projectile after the collision [16], and charge exchange [17]. Unfortunately, the atomic physics experiments in the energy region around $E=1 \mathrm{GeV} / \mathrm{u}$ supply only total cross sections $[18,19]$. More selective differential cross sections with respect to the impact parameter or the ion scattering angle are still missing.

In Sec. II we present the formalism of the coupledchannel equations that we used throughout our calculations. The evaluation of the potential matrix elements and their symmetries are emphasized. The subsequent section treats the numerical results, in particular the comparison of the outcome of perturbation theory, with results of the coupled-channel calculations for ionization and pair creation. The question of gauge invariance is considered in Sec. IV. Finally, Sec. V contains a brief summary.

\section{THEORY}

In order to calculate electron excitation and ionization as well as pair creation, we want to solve the time- 
dependent Dirac equation

$$
i \frac{\partial}{\partial t} \psi(\mathbf{r}, t)=\hat{H}(\mathbf{R}(t)) \psi(\mathbf{r}, t),
$$

where $\mathbf{R}(t)$ denotes the time-dependent position of the projectile. Here we have used the semiclassical approximation (SCA) [20-22], where the nuclei are assumed to move on classical trajectories.

Equation (1) is a partial differential equation in four dimensions. The direct solution requires considerable numerical effort. In central heavy-ion collisions the cylindrical symmetry reduces the problem to two spatial coordinates. For excitation and ionization such a calculation has been performed by Becker et al. [23], where the differential operators were replaced by finite differences. An extension to pair creation is presented by Thiel et al. [24]. One difficulty in performing this procedure is introduced by the finite grid size and by the finite number of grid points.

Here we will apply a different method that has already been employed for nonrelativistic collisions. The wave function is expanded into a complete basis set

$$
\psi_{i}(\mathbf{r}, t)=\oiint_{k} a_{k i}(t) \phi_{k}(\mathbf{r}) \exp \left(-i E_{k} t\right) .
$$

The subscript $i$ of the wave function indicates different possible initial conditions:

$$
a_{k i}(t \rightarrow-\infty)=\delta_{k i}
$$

Usually, the total Hamiltonian is split into two contributions $\hat{H}=\hat{H}_{0}+\hat{V}$. Choosing the eigenstates of the unperturbed Hamiltonian $\hat{H}_{0}$ as basis states, i.e.,

$$
\hat{H}_{0} \phi_{k}=E_{k} \phi_{k},
$$

the basis wave functions are orthonormal

$$
\left\langle\phi_{f} \mid \phi_{k}\right\rangle=\delta_{f k} \text {. }
$$

For very fast collisions we will employ the atomic basis. The unperturbed Hamiltonian consists only of the kinetic energy and of the target potential

$$
\hat{H}_{0}=\hat{\boldsymbol{\alpha}} \cdot \hat{\mathbf{p}}+\hat{\beta}+V_{T} .
$$

The projectile potential is thought of as the perturbing potential $\hat{V} . \hat{H}_{0}$ is independent of time and so are the basis states. Consequently, only the matrix elements of the projectile potential $V_{p}$ result in transitions between the states and we obtain for the coupled-channel equations

$$
\dot{a}_{f i}=-i \sum_{k} a_{k i}(t)\left\langle\phi_{f}|\hat{V}| \phi_{k}\right\rangle \exp \left[i\left(E_{f}-E_{k}\right) t\right] .
$$

The basis contains only target-centered states, which do not allow for the description of charge exchange. To take also these channels into account requires a basis set extended by projectile-centered states. But target- and projectile-centered states are not orthonormal to each other and therefore a modified form of the coupled channels has to be utilized [4]. In this paper we will not investigate charge-exchange processes.

For weak potentials $\widehat{V}$ perturbation theory might be a reliable tool. In first-order perturbation theory the amplitude after the collision $(t \rightarrow \infty)$ reads

$$
a_{f i}(t \rightarrow \infty)=-i \int_{-\infty}^{\infty} d t\left\langle\phi_{f}|\hat{V}| \phi_{i}\right\rangle \exp \left[i\left(E_{f}-E_{i}\right) t\right]
$$

Since the potential depends linearly on the projectile charge $Z_{p}$, the perturbative probabilities and cross sections depend quadratically on the projectile charge.

For our calculations it is very important that unitarity remains conserved during the time evolution. This is not fulfilled in perturbation theory. Thus it may happen that perturbation theory results in excitation probabilities greater than unity, which is physically meaningless.

A Hermitian potential matrix, i.e., $V_{f i}^{*}=V_{i f}$, guarantees the conservation of probability in the framework of coupled-channel calculations. Even the orthonormality of the wave functions remains conserved during the time evolution.

\section{A. Projectile potential}

In order to solve the coupled-channel equations (7) we need the matrix elements $\left\langle\phi_{f}|\hat{V}| \phi_{k}\right\rangle$. First we want to determine the projectile potential. In relativistic heavyion collisions a straight line is a good approximation for the trajectory of the projectile $[25,26]$. Likewise, we may assume that the target remains fixed at the origin of the coordinate system.

The coordinate system is chosen so that the projectile moves parallel to the $z$ axis and the $x$ - $z$ plane is the scattering plane (see Fig. 2). The projectile potential as seen by an observer in the target system is determined by a Lorentz transformation. In the inertial system of the projectile the projectile potential is simply a Coulomb potential

$$
\mathbf{A}^{\prime}=0, \quad A^{0 \prime}=\frac{Z_{P} e^{2}}{\widetilde{r}^{\prime}},
$$

where

$$
\widetilde{r}^{\prime}=\left[(x-b)^{2}+y^{2}+\gamma^{2}\left(z-v_{P} t\right)^{2}\right]^{1 / 2}
$$

denotes the distance from the projectile as seen in the projectile system. Primed quantities are measured in the projectile system. The transformation of the potentials (9) into the target frame yields the Liénard-Wiechertpotentials [27]

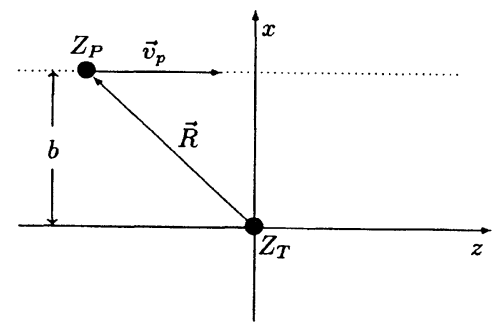

FIG. 2. Coordinate system used in our calculations. The projectile moves within the $x-z$ plane along a straight line with distance $b$ parallel to the $z$ axis. 


$$
A^{0}=+\gamma \frac{Z_{P} e^{2}}{\widetilde{r}^{\prime}}, \quad A^{1}=A^{2}=0, \quad A^{3}=-\gamma v_{P} \frac{Z_{P} e^{2}}{\widetilde{r}^{\prime}}
$$

For completeness we write the total Hamiltonian

$$
\begin{aligned}
\hat{\boldsymbol{H}} & =\hat{\boldsymbol{\alpha}} \cdot \hat{\mathbf{p}}+\hat{\beta}+V_{T}+\hat{\boldsymbol{V}}_{P} \\
& =\hat{\boldsymbol{\alpha}} \cdot \hat{\mathbf{p}}+\hat{\boldsymbol{\beta}}-\frac{Z_{T} e^{2}}{r}+\gamma \frac{Z_{P} e^{2}\left(v_{P} \hat{\alpha}_{3}-1\right)}{\widetilde{\boldsymbol{r}}^{\prime}} .
\end{aligned}
$$

For the target we assumed the potential of a fully ionized pointlike nucleus with the charge $Z_{T}$. Equivalently we can employ a screened target potential.

For the calculation of the matrix elements the quantity

$$
\frac{1}{\widetilde{r}^{\prime}}=\frac{1}{\left[(x-b)^{2}+y^{2}+\gamma^{2}\left(z-v_{P} t\right)^{2}\right]^{1 / 2}}=\frac{1}{\left|\mathbf{r}^{\prime}-\mathbf{R}^{\prime}(t)\right|}
$$

is expanded into a multipole series. The electron and nuclear coordinates are $\mathbf{r}^{\prime}=(x, y, \gamma z)$ and $\mathbf{R}^{\prime}=\left(b, 0, \gamma v_{p} t\right)$, respectively. The components of $\mathbf{r}^{\prime}$ in spherical polar coordinates read

$$
\begin{aligned}
& r^{\prime}=\left|\mathbf{r}^{\prime}\right|=r\left(\sin ^{2} \theta+\gamma^{2} \cos ^{2} \theta\right)^{1 / 2}=r h(\theta), \\
& \cos \theta^{\prime}=\frac{z^{\prime}}{r^{\prime}}=\frac{\gamma \cos \theta}{h(\theta)} .
\end{aligned}
$$

The function $h(\theta)$ is defined by Eq. (14). Now the multipole expansion reads

$$
\begin{aligned}
\frac{1}{\left|\mathbf{r}^{\prime}-\mathbf{R}^{\prime}\right|} & =\sum_{l=0}^{\infty} \frac{r_{<}^{\prime l}}{r_{>}^{\prime l+1}} P_{t}(\cos \theta) \\
& =\left\{\begin{array}{l}
\sum_{l=0}^{\infty} \frac{4 \pi}{2 l+1} \frac{r^{l} h^{l}(\theta)}{R^{\prime l+1}} \sum_{m=-l}^{l} Y_{l m}^{*}\left(\hat{\mathbf{R}}^{\prime}\right) Y_{l m}\left(\hat{\mathbf{r}}^{\prime}\right), \quad r^{\prime} \leq R^{\prime} \\
\sum_{l=0}^{\infty} \frac{4 \pi}{2 l+1} \frac{R^{\prime l}}{r^{l+1} h^{l+1}(\theta)} \sum_{m=-l}^{l} Y_{l m}^{*}\left(\hat{\mathbf{R}}^{\prime}\right) Y_{l m}\left(\hat{\mathbf{r}}^{\prime}\right), \quad r^{\prime} \geq R^{\prime}
\end{array}\right. \\
& =\sum_{l=0}^{\infty} \frac{4 \pi}{2 l+1} g_{l}(r, \theta) \sum_{m=-l}^{l} Y_{l m}^{*}\left(\hat{\mathbf{R}}^{\prime}\right) Y_{l m}\left(\hat{\mathbf{r}}^{\prime}\right),
\end{aligned}
$$

where $\hat{\mathbf{R}}^{\prime}$ and $\hat{\mathbf{r}}^{\prime}$, respectively, denote the directions of the vectors $\mathbf{R}^{\prime}$ and $\mathbf{r}^{\prime}$. The function $g_{l}$ is defined by

$$
g_{l}(r, \theta)= \begin{cases}\frac{r^{l} h^{l}(\theta)}{R^{\prime l+1}} & \text { if } r^{\prime}=r h(\theta)<R^{\prime} \\ \frac{R^{\prime l}}{r^{l+1} h^{l+1}(\theta)} & \text { if } r^{\prime}=r h(\theta)>R^{\prime} .\end{cases}
$$

\section{B. Matrix elements}

In this subsection the matrix elements $\left\langle\phi_{f}|\hat{V}| \phi_{i}\right\rangle$ will be evaluated. Due to the spherical symmetric target potential the atomic basis functions can be represented in the form

$$
\phi(\mathbf{r})=\frac{1}{r}\left(\begin{array}{cc}
u(r) & \chi_{\kappa}^{\mu}(\Omega) \\
i v(r) & \chi_{-\kappa}^{\mu}(\Omega)
\end{array}\right),
$$

with real radial functions $u(r)$ and $v(r)$. The continua are discretized by the use of relativistic wave packets [28]

$$
\psi_{k}(r)=\frac{1}{\sqrt{\Delta E}} \int_{E_{k}-\Delta E / 2}^{E_{k}+\Delta E / 2} \psi(r, E) d E,
$$

where $\psi(r, E)$ is the exact continuum eigenstate of $\hat{H}_{0}$ for the energy $E$. Since many continuum wave functions $\psi(r, E)$ with different wavelengths interfere, the wave packets $\psi_{k}(r)$ fall off faster as a function of $r$.

First we want to consider the electric matrix elements. After inserting the multipole expansion (16), except for the factor $\left(-\gamma Z_{p} e^{2}\right)$, the matrix elements read

$$
\left\langle\phi_{f}\left|\frac{1}{\left|\mathbf{r}^{\prime}-\mathbf{R}^{\prime}\right|}\right| \phi_{i}\right\rangle=\int_{0}^{\infty} d r r^{2} \int d \Omega \frac{1}{r}\left(u_{f} \chi_{\kappa_{f}}^{\mu_{f}^{\dagger}},-i v_{f} \chi_{-\kappa_{f}}^{\mu_{f}^{\dagger}}\right) \sum_{L} \frac{4 \pi}{2 L+1} g_{L}(r, \theta) \sum_{M} Y_{L M}^{*}\left(\widehat{\mathbf{R}}^{\prime}\right) Y_{L M}\left(\hat{\mathbf{r}}^{\prime}\right) \frac{1}{r} \sum_{i v_{i}}^{u_{-} \chi_{-\kappa_{i}}} \chi_{\kappa_{i}}^{\mu_{i}}
$$

with $g_{L}$ defined by (17). Choosing the $x-z$ plane as the scattering plane the azimuthal angle of $\widehat{\mathbf{R}}^{\prime}$ is zero and the complex conjugation of the corresponding spherical harmonic may be omitted.

Originally the multipoles were defined on the surface of the unit sphere in the projectile frame. Since the basis wave functions are defined in the target system, we transformed the multipoles into the same system. This leads to a Lorentz contraction of the unit sphere and thus the multipoles are now defined on the surface of an oblate ellipsoid in the target frame. Consequently, the transition from the inner region of the multipole expansion to the outer region takes place on this ellipsoid surface. This situation is depicted in Fig. 3. 


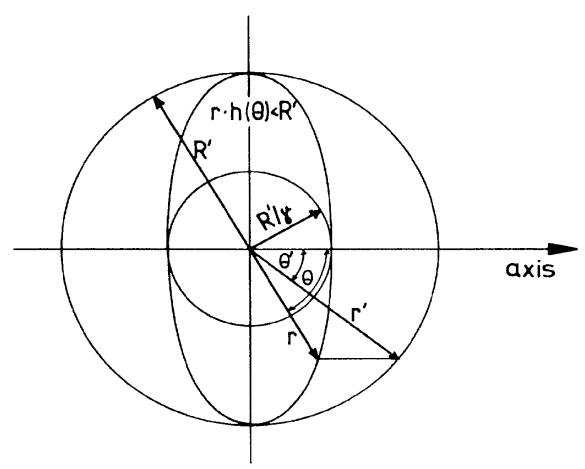

FIG. 3. Depicted are different regions of the multipole expansion. The boundary between both regions is located on the marked ellipsoid. On this boundary the explicit form of the multipole expansion changes. The inner region inside the sphere with radius $R^{\prime} / \gamma$ is indicated as well as the outer region outside the sphere with radius $R^{\prime}$. In these two cases the classification is independent of the polar angle, while in the region between these radii it depends on the angle $\theta$.

The boundary between both regions is at $r=R^{\prime} / h(\theta)$ and thus varies between the values $r=R^{\prime} / \gamma$ and $r=R^{\prime}$. Therefore inside the sphere with radius $R^{\prime} / \gamma$ we areindependent of the angle - in the inner region, while outside the sphere with radius $R^{\prime}$ we are always in the outer region. Only between these radii does the classification depend on the polar angle.

Due to the distinction in the definition of the function $g_{L}$ the radial and the angular integration do not separate in the intermediate region. This is different from the nonrelativistic case. Thus in this region a twodimensional integral has to be computed. $g_{L}(r, \theta)$ factorizes in the regions $r<R^{\prime} / \gamma$ as well as $r>R^{\prime}$ and thus the integrations separate here.

We rewrite the matrix element

$$
\left\langle\phi_{f}\left|\frac{1}{\left|\mathbf{r}^{\prime}-\mathbf{R}^{\prime}\right|}\right| \phi_{i}\right\rangle=\sum_{L=0}^{\infty} \sum_{M} \frac{4 \pi}{2 L+1} Y_{L m}\left(\hat{\mathbf{R}}^{\prime}\right) \mathcal{S}_{f i, L M}^{\phi}\left(R^{\prime}\right),
$$

with

$$
S_{f i, L M}^{\phi}\left(R^{\prime}\right)=\int_{0}^{\infty} d r \mathscr{R}(r) \mathcal{W}_{\kappa_{f} \kappa_{i} L M}^{\phi}(r) .
$$

Here the abbreviation

$$
\mathcal{R}(r)=u_{f}(r) u_{i}(r)+v_{f}(r) v_{i}(r)
$$

and the angular integral

$$
\begin{aligned}
\mathcal{W}_{\kappa_{f} \kappa_{i} L M}^{\phi}(r) & =\int d \Omega g_{L}(r, \theta) \chi_{\kappa_{f}}^{\mu_{f}^{\dagger}} Y_{L M}\left(\hat{\mathbf{r}}^{\prime}\right) \chi_{\kappa_{i}}^{\mu_{i}} \\
& =\int d \Omega w_{\kappa_{f} \kappa_{i} L M}^{\phi}(r, \theta)
\end{aligned}
$$

have been introduced. In (24) we defined the angular function $w_{\kappa_{f} \kappa_{i} L M}^{\phi}$, which is given by

$$
\begin{aligned}
w_{\kappa_{f} \kappa_{i} L M}^{\phi}(r, \theta)=\sum_{m} & \left(l_{f}, \frac{1}{2}, j_{f} \mid \mu_{f}-m, m, \mu_{f}\right) \\
& \times\left(l_{i}, \frac{1}{2}, j_{i} \mid \mu_{i}-m, m, \mu_{i}\right) \\
& \times Y_{l_{f}, \mu_{f}-m}^{*}(\theta, \varphi) Y_{l_{i}, \mu_{i}-m}(\theta, \varphi) \\
& \times Y_{L M}\left(\theta^{\prime}, \varphi^{\prime}\right) g_{L}(r, \theta)
\end{aligned}
$$

The integration over $\varphi$ yields the usual addition rule for the magnetic quantum numbers, which is well known from the nonrelativistic description. Therefore, in Eq. (21) the sum over $M$ may be omitted when $M=\mu_{f}-\mu_{i}$ is substituted. Also, the parity fulfills the same selection rule as in the nonrelativistic case. The parity of $Y_{L M}\left(\theta^{\prime}, \varphi\right)$ equals the parity of $Y_{L M}(\theta, \varphi)$, since $\theta^{\prime}(\pi-\theta)=\pi-\theta^{\prime}(\theta)$. In addition, $h(\theta)$ has even parity: $h(\pi-\theta)=h(\theta)$. It follows that the integrals over the angle vanish if $l_{f}+l_{i}+L=0(\bmod 2)$. Otherwise it is sufficient to integrate from 0 to $\pi / 2$ and to double the integral afterwards.

It is worthwhile to note that even in the regions $r<R^{\prime} / \gamma$ and $r>R^{\prime}$, where the integrals separate, the standard triangle rule is violated in the relativistic case. In the nonrelativistic case only those $L$ values contribute that fulfill $\left|j_{f}-j_{i}\right| \leq L \leq\left|j_{f}+j_{i}\right|$. On the other hand, for relativistic velocities all values $L=0 \ldots \infty$ contribute [29].

Now we turn the discussion to the analogous expressions for the magnetic matrix elements

$$
\begin{aligned}
\left\langle\phi_{f}\left|\frac{\hat{\alpha}_{3}}{\left|\mathbf{r}^{\prime}-\mathbf{R}^{\prime}\right|}\right| \phi_{i}\right\rangle=i \sum_{L=0}^{\infty} \sum_{M=-L}^{+L} & \frac{4 \pi}{2 L+1} \\
& \times Y_{L M}\left(\hat{\mathbf{R}}^{\prime}\right) g_{f i, L M}^{\mathbf{A}}\left(R^{\prime}\right)
\end{aligned}
$$

with

$$
g_{f i, L M}^{\mathrm{A}}\left(R^{\prime}\right)=\int_{0}^{\infty} d r\left(u_{f} v_{i} \mathcal{W}_{\kappa_{f},-\kappa_{i} L M}^{\mathrm{A}}-v_{f} u_{i} \mathcal{W}_{-\kappa_{f}, \kappa_{i} L M}^{\mathrm{A}}\right)
$$

Note that in contrast to the electric matrix elements, the matrix $\hat{\alpha}_{3}$ couples upper and lower components of the spinors. This leads to the sign combinations of the $\kappa$ subscripts of $\mathcal{W}^{\mathrm{A}}$. These quantities are defined by the angular integral

$$
\begin{aligned}
\mathcal{W}_{\kappa_{f}, \kappa_{i} L M}^{\mathbf{A}}(r) & =\int d \Omega g_{L}(r, \theta) \chi_{\kappa_{f}}^{\mu_{f}^{\dagger}} Y_{L M}\left(\hat{\mathbf{r}}^{\prime}\right) \hat{\sigma}_{3} \chi_{\kappa_{i}}^{\mu_{i}} \\
& =\int d \Omega w_{\kappa_{f}, \kappa_{i} L M}^{\mathbf{A}}(r, \theta)
\end{aligned}
$$

with the corresponding angular function

$$
\begin{aligned}
w_{\kappa_{f}, \kappa_{i} L M}^{\mathbf{A}}(r, \theta) & =\sum_{m} 2 m\left(l_{f}, \frac{1}{2}, j_{f} \mid \mu_{f}-m, m, \mu_{f}\right) \\
& \times\left(l_{i}, \frac{1}{2}, j_{i} \mid \mu_{i}-m, m, \mu_{i}\right) \\
& \times Y_{l_{f}, \mu_{f}-m}^{*}(\theta, \varphi) Y_{l_{i}, \mu_{i}-m}(\theta, \varphi) \\
& \times Y_{L M}\left(\theta^{\prime}, \varphi^{\prime}\right) g_{L}(r, \theta)
\end{aligned}
$$

The factor $2 m$ results from the relation 


$$
\chi_{m^{\prime}}^{\dagger} \hat{\sigma}_{3} \chi_{m}=2 m \delta_{m^{\prime} m} \text {. }
$$

In addition, due to the Kronecker $\delta$ the sum over $m^{\prime}$ could be easily performed.

We deduce from Eqs. (21) and (26) that the electric matrix element is real, while the magnetic matrix element is purely imaginary:

$$
V_{f i}=\operatorname{Re}\left(V_{f i}\right)+i \operatorname{Im}\left(V_{f i}\right)=V_{f i}^{\phi}+V_{f i}^{\mathrm{A}} .
$$

Finally, we want to discuss some symmetry relations of the matrix elements. The explicit use of these symmetry relations considerably lowers the computational effort and the storage requirements for the matrix elements.

First we study the behavior of $\mathcal{S}_{f i, L M}^{\phi}$ when exchanging $i \leftrightarrow f$,

$\mathcal{S}_{i f, L \mu_{i}-\mu_{f}}^{\phi}\left(R^{\prime}\right)=\int d r \mathcal{R}(r) \int d \Omega w_{\kappa_{i} \kappa_{f}, L, \mu_{i}-\mu_{f}}^{\phi}$.

Using the symmetry relations of the Clebsch-Gordan coefficients and of the spherical harmonics contained in (25), we derive

$$
\mathcal{S}_{i f, L \mu_{i}-\mu_{f}}^{\phi}=(-1)^{\mu_{i}-\mu_{f}} \mathcal{S}_{f i, L \mu_{f}-\mu_{i}}^{\phi} .
$$

The analogous result for the magnetic matrix elements reads

$$
\mathcal{S}_{i f, L \mu_{i}-\mu_{f}}^{\mathbf{A}}=(-1)^{\mu_{i}-\mu_{f}+1} \mathcal{G}_{f i, L \mu_{f}-\mu_{i}}^{\mathbf{A}} .
$$

These expressions may be inserted into the expansions (21) and (26) and we obtain

$$
\left\langle\phi_{i}\left|\frac{1}{\left|\mathbf{r}^{\prime}-\mathbf{R}^{\prime}\right|}\right| \phi_{f}\right\rangle=\left\langle\phi_{f}\left|\frac{1}{\left|\mathbf{r}^{\prime}-\mathbf{R}^{\prime}\right|}\right| \phi_{i}\right\rangle
$$

and

$$
\left\langle\phi_{i}\left|\frac{\hat{\alpha}_{3}}{\left|\mathbf{r}^{\prime}-\mathbf{R}^{\prime}\right|}\right| \phi_{f}\right\rangle=-\left\langle\phi_{f}\left|\frac{\hat{\alpha}_{3}}{\left|\mathbf{r}^{\prime}-\mathbf{R}^{\prime}\right|}\right| \phi_{i}\right\rangle .
$$

With (31) we conclude for the total matrix element

$$
V_{i f}=\operatorname{Re}\left(V_{i f}\right)+i \operatorname{Im}\left(V_{i f}\right)=\operatorname{Re}\left(V_{f i}\right)-i \operatorname{Im}\left(V_{f i}\right)=V_{f i}^{*} .
$$

Since $\hat{V}$ is a Hermitian operator, this result is quite reasonable.

One additional symmetry is provided by simultaneously changing the sign of the magnetic quantum numbers:

$$
\left(\mu_{i}, \mu_{f}\right) \leftrightarrow\left(-\mu_{i},-\mu_{f}\right) .
$$

Only the angular functions $w_{\kappa_{f} \kappa_{i}, L M}^{\phi}$ and $w_{\kappa_{f} \kappa_{i}, L M}^{\mathrm{A}}$ contain the magnetic quantum numbers via the ClebschGordan coefficients. The angular functions corresponding to the changed quantum numbers according to (38) in the following are marked by a tilde. For the transformed angular functions we find

$$
\begin{aligned}
\widetilde{w}_{\kappa_{f} \kappa_{i} L,-\mu_{f}+\mu_{i}=}^{\phi}=\sum_{m}(-1)^{l_{f}+(1 / 2)+j_{f}}\left(l_{f}, \frac{1}{2}, j_{f} \mid \mu_{f}-m, m, \mu_{f}\right)(-1)^{l_{i}+(1 / 2)+j_{i}}\left(l_{i}, \frac{1}{2}, j_{i} \mid \mu_{i}-m, m, \mu_{i}\right) \\
\quad \times(-1)^{\mu_{f}-m} Y_{l_{f} \mu_{f}-m}^{*}(\theta, \varphi)(-1)^{\mu_{i}-m} Y_{l_{i} \mu_{i}-m}(\theta, \varphi)(-1)^{\mu_{i}-\mu_{f}} Y_{L \mu_{f}-\mu_{i}}\left(\theta^{\prime}, \varphi\right) g_{L}(r, \theta) .
\end{aligned}
$$

Combining all signs and using

$$
\begin{aligned}
j & =|\kappa|-\frac{1}{2}, \\
l & = \begin{cases}-\kappa-1 & (\kappa<0) \\
\kappa & (\kappa>0)\end{cases} \\
& =|\kappa|-\frac{1}{2}+\frac{1}{2} \operatorname{sgn} \kappa,
\end{aligned}
$$

results in

$$
\widetilde{w}_{\kappa_{f} \kappa_{i} L,-\mu_{f}+\mu_{i}}^{\phi}=(-1)^{\left(\operatorname{sgn} \kappa_{f}+\operatorname{sgn} \kappa_{i}\right) / 2+1} w_{\kappa_{f} \kappa_{i} L, \mu_{f}}^{\phi}-\mu_{i}
$$

and hence,

$$
\widetilde{\mathcal{S}}_{\kappa_{f} \kappa_{i} L,-\mu_{f}+\mu_{i}}^{\phi}=(-1)^{\left(\operatorname{sgn} \kappa_{f}+\operatorname{sgn} \kappa_{i}\right) / 2+1} \mathcal{g}_{\kappa_{f} \kappa_{i} L, \mu_{f}-\mu_{i}}^{\phi} .
$$

For the magnetic matrix elements the analogous expression reads

$$
\widetilde{w}_{\kappa_{f} \kappa_{i} L,-\mu_{f}+\mu_{i}}^{\mathbf{A}}=(-1)^{\left(\operatorname{sgn} \kappa_{f}+\operatorname{sgn} \kappa_{i}\right) / 2} w_{\kappa_{f} \kappa_{i} L, \mu_{f}-\mu_{i}}^{\mathbf{A}} .
$$

From Eq. (27) we know that $g^{\mathbf{A}}$ contains the functions $w^{\mathbf{A}}$ with negative $\kappa_{i}$ or $\kappa_{f}$. This minus sign may be pulled out and leads to an additional minus sign:

$$
\widetilde{\mathcal{S}}_{\kappa_{f} \kappa_{i} L,-\mu_{f}+\mu_{i}}^{\mathbf{A}}=(-1)^{\left(\operatorname{sgn} \kappa_{f}+\operatorname{sgn} \kappa_{i}\right) / 2+1} \mathcal{G}_{\kappa_{f} \kappa_{i} L, \mu_{f}-\mu_{i}}^{\mathbf{A}} .
$$

Finally we consider the operation of time reversal: $t \rightarrow-t$. Using this symmetry it is possible to calculate and to store only the matrix elements for the incoming projectile trajectory. Only the spherical harmonic $Y_{L M}\left(\hat{\mathbf{R}}^{\prime}\right)=Y_{L M}\left(\theta_{R^{\prime}}\right)$ in Eqs. (21) and (26) are influenced by time reversal. Applying time reversal the spherical harmonic transforms according to $Y_{L M}\left(\pi-\theta_{R^{\prime}}\right)$ $=(-1)^{L-M} Y_{L M}\left(\hat{\mathbf{R}}^{\prime}\right)$. For all contributing $L$ the value of $(-1)^{L-M}$ is the same due to $L=l_{f}-l_{i}(\bmod 2)$. With $M=\mu_{f}-\mu_{i}$, we finally get

$$
\operatorname{Re}\left[V_{f i}(-t)\right]=(-1)^{l_{f}-l_{i}-\left(\mu_{f}-\mu_{i}\right)} \operatorname{Re}\left[V_{f i}(t)\right] .
$$

Again the calculation is analogous for the magnetic matrix element. Because of $L=l_{f}-l_{i}+1(\bmod 2)$ an additional minus sign arises:

$\operatorname{Im}\left[V_{f i}(-t)\right]=(-1)^{l_{f}-l_{i}+1-\left(\mu_{f}-\mu_{i}\right)} \operatorname{Im}\left[V_{f i}(t)\right]$.

The results for the electric and magnetic matrix elements may be combined to

$$
V_{f i}(-t)=(-1)^{l_{f}-l_{i}+\mu_{f}-\mu_{i}} V_{f i}^{*}(t)
$$




\section{Adiabatic initial conditions}

The coupled-channel equations (7) have to be solved with the initial conditions (3). For the numerical solution we employ the method of Shampine and Gordon [30], which is a modified Adams-Moulton predictor-corrector method of variable order with variable step size.

For test purposes we solved the coupled-channel equations for the system $\mathrm{Pb}-\mathrm{Pb}$ with the bombarding energy $E=500 \mathrm{MeV} / \mathrm{u}$ and impact parameter $b=100 \mathrm{fm}$. The basis consists of the 10 deepest bound states, i.e., the $K$ and $L$ shell, and 60 continuum states. As an initial condition we consider a single electron in the $1 s$ state with $\mu=-\frac{1}{2}$. The result of this calculation is represented in Fig. 4(a). Just after the beginning of the integration the main contribution to the continuum channels stems from the state with $E=1.1, \kappa=-2, \mu=-\frac{1}{2}$. Later on the probability for the electron to be in the initial state decreases while the probability for the electron to be excited or to be ionized increases. The excitations are maximal around the distance of closest approach at $t \approx 0$. The occupation probabilities during the collision are not observable experimentally. Only the occupations for $t \rightarrow \infty$ are observable, e.g., by measuring the energy distribution of the $\delta$ electrons.

Considering Fig. 4(a) strong oscillations of the occupation probabilities for $t<0$ are noticed. These are due to

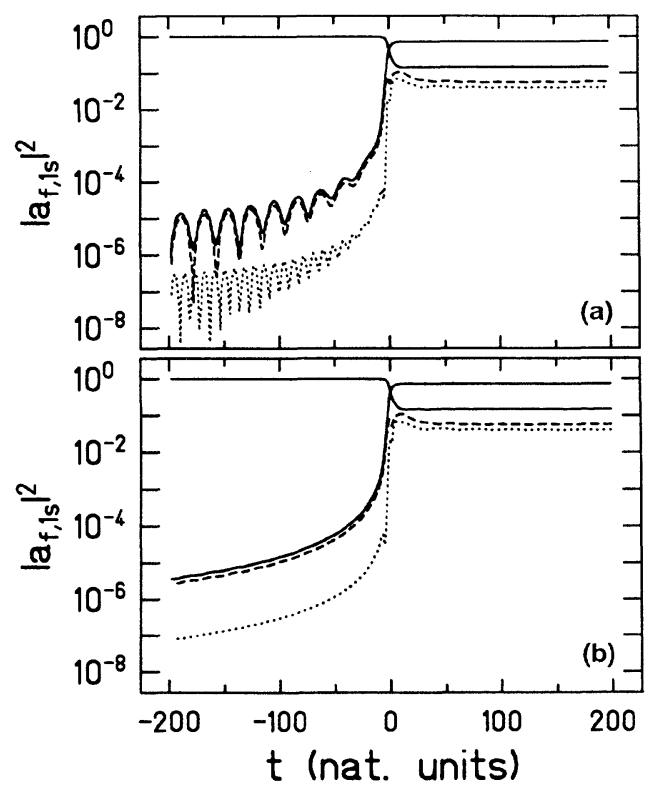

FIG. 4. Solution of the coupled-channel equations for the system $\mathrm{Pb}-\mathrm{Pb}$ at a bombarding energy $E=500 \mathrm{MeV} / \mathrm{u}$ and impact parameter $b=100 \mathrm{fm}$. The squared absolute values of the amplitudes $\left|a_{f, 1 s}\right|^{2}$ are shown as a function of the collision time $t$. $t$ is given by multiples of the unit $1.29 \times 10^{-21} \mathrm{sec}$. Note the strong excitation around the distance of closest approach at $t \approx 0$. Full lines: $1 s$ state and sum over all continuum states, respectively; dashed line: continuum state with $E=1.1, \kappa=-2$, $\mu=-\frac{1}{2}$; dotted line: continuum state with $E=1.5, \kappa=-2$, $\mu=-\frac{1}{2}$. (a) Initial condition $a_{f i}=\delta_{f i}$. (b) Using adiabatic initial conditions the oscillations almost disappear. the fact that the numerical integration starts at the finite time $-T_{0} \neq-\infty$, when the projectile is far away. In Fig. 5 we depict the complex probability amplitude during a short period after $-T_{0}$ for the dominant continuum states with $E=1.1$ and $\kappa=-2, \mu=-\frac{1}{2}$. The amplitude follows a spiral curve starting at the origin.

For this early time the projectile is far away and the interaction is very weak. Thus we can apply perturbation theory; i.e., we set $a_{k i} \approx \delta_{k i}$. Now the coupled-channel equations simply read

$$
\dot{a}_{f i}=-i\left\langle\phi_{f}|\hat{V}| \phi_{i}\right\rangle \exp \left[i\left(E_{f}-E_{i}\right) t\right] .
$$

Initially, the matrix element changes very slowly in time and we may consider it to be constant. Hence Eq. (49) can be integrated and for $f \neq i$ we get

$$
\begin{aligned}
a_{f i}(t)=\frac{-M_{f i}}{E_{f}-E_{i}} & \left\{\exp \left[i\left(E_{f}-E_{i}\right) t\right]\right. \\
& \left.-\exp \left[-i\left(E_{f}-E_{i}\right) T_{0}\right]\right\} .
\end{aligned}
$$

The time-dependent phase factor leads to a circular motion in the complex plane, which is broadened by the slowly increasing matrix element. The additional term in the curly brackets guarantees the initial condition $a_{f i}\left(t=-T_{0}\right)=0$. But just this term is responsible for the shift of the origin of the complex plane from the midpoint of the spiral curve to the starting point. Calculating the squared absolute value of the complex amplitude, the value decreases after every revolution because the starting point is nearly reached. The frequency of these oscillations equals the energy difference $\omega=E_{f}-E_{i}$.

This is clearly seen for the depicted continuum states in Fig. 4(a). Taking into account the energy of the $1 s$ state, $E_{i} \approx 0.8 \mathrm{mc}^{2}$, we get $T=2 \pi / \omega \approx 21$ and $T \approx 9$, respectively for the cycle time of the oscillations. This is in fair agreement with the results as displayed in Fig. 4(a).

Instead of suddenly turning on the potential at

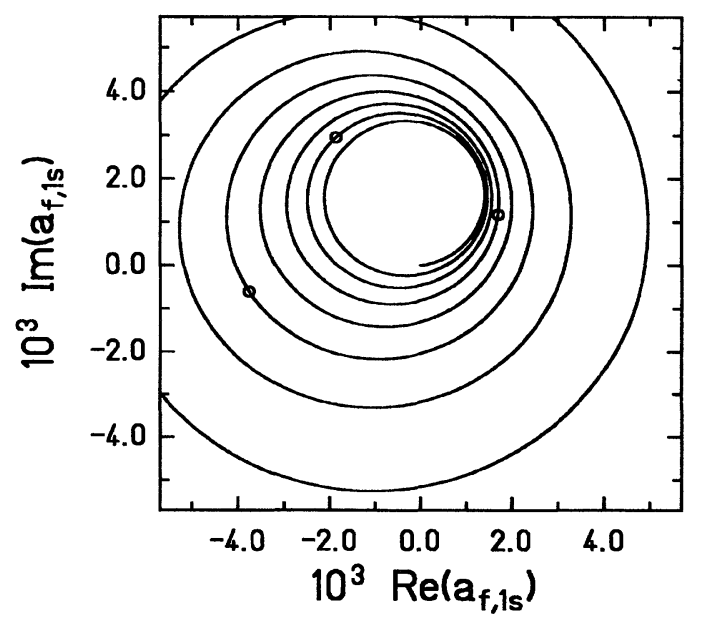

FIG. 5. Complex probability amplitude for the continuum state with $E=1.1$ and $\kappa=-2, \mu=-\frac{1}{2}$ for the same system as in Fig. 4. The spiraling curve results from the complex phase factor in the coupled-channel equations. 
$t=-T_{0}$ we now assume that the interaction has increased very slowly, i.e., adiabatically, from zero in such a way that a single spiral revolution cannot be distinguished from a true circle. In this case the origin of the complex plane and the midpoints of the circles coincide and thus the term $\exp \left[-i\left(E_{f}-E_{i}\right) T_{0}\right]$ has to be omitted. Hence the adiabatic initial conditions read

$$
\begin{aligned}
a_{f i}\left(t=T_{0}\right) & =\frac{-M_{f i}}{E_{f}-E_{i}} \exp \left[i\left(E_{f}-E_{i}\right) T_{0}\right] \\
& =\frac{1}{i\left(E_{f}-E_{i}\right)} \dot{a}_{f i} .
\end{aligned}
$$

Afterwards the total probability has to be normalized to unity.

In Fig. 4(b) the outcome of a coupled-channel calculation using the adiabatic initial conditions is displayed. It is the same calculation as in Fig. 4(a), but using the initial conditions (51). Apparently the oscillations almost disappeared. Nevertheless, the probabilities for $t \rightarrow \infty$ remain almost unchanged by these initial conditions. Of course, the shift of the starting point of the integration toward earlier times results in a similar disappearance of these oscillations. By the use of adiabatic initial conditions we avoid the calculation of the corresponding matrix elements.

\section{NUMERICAL RESULTS}

The methods as described in the preceding section will be applied to physical problems of current interest. We calculate ionization and pair-creation probabilities in relativistic heavy-ion collisions. In particular we compare the results of coupled-channel calculations with the outcome of perturbation theory.

In order to calculate ionization probabilities and cross sections we have to solve the coupled-channel equations using a basis that contains bound states as well as continuum states. At a first glance it seems that the influence of the states of the negative-energy continuum is negligible. But it turns out that there can be clear differences between calculations that neglect the negative-energy states and calculations using a basis enlarged by the negativeenergy continuum.

We start our investigations by considering the ionization of a $K$-shell electron. For this purpose the coupledchannel equations (7) are solved with the initial conditions

$$
a_{k, 1 s}(t \rightarrow-\infty)=\delta_{k, 1 s}
$$

or with the adiabatic initial conditions.

The ionization probability is just the probability for the electron to be after the collision in one of the continuum channels of positive energy, thus [31-33]

$$
P_{K}(b)=\sum_{E_{k}>1}\left|a_{k, 1 s}(t \rightarrow \infty)\right|^{2} .
$$

Usually the $K$ shell is occupied by two electrons. Thus, the mean number of electrons ionized per collision from the $K$ shell can be calculated by simply multiplying the probability $(53)$ by 2 . Since up to two electrons may be ionized in a single collision, this is no longer a probability normalized to unity.

These considerations are only true if the target is totally ionized except for the $K$ shell. If not, we have to calculate the probability for the coincident observation of the ionized electron with the hole in the $K$ shell. This correlated probability is given in [32,33].

In Fig. 6 we show a comparison between results from first-order perturbation theory and coupled-channel calculations. It concerns the collision system $\mathrm{Pb}^{81+}+Z_{p}$ with projectile charges up to the end of the periodic system. As already mentioned, the ionization probability in perturbation theory scales with $Z_{p}^{2}$. In order to facilitate the comparison the probabilities were divided by $Z_{p}^{2}$. Therefore the ionization probabilities given by perturbation theory are simply represented by horizontal straight lines.

Apparently the results of the coupled-channel equations approach continuously the perturbative result for $Z_{p} \rightarrow 0$. For larger nuclear charges the ionization probability increases first over the perturbative probability and reaches a maximum at $Z_{p} \approx 50$. For even larger $Z_{p}$ the probability decreases again.

The proportionality of the perturbative probability with $Z_{p}^{2}$ for sufficiently large $Z_{p}$ leads to a violation of unitarity, i.e., to probabilities larger than unity. However, unitarity is conserved in coupled-channel calculations. Thus for large projectile charges the probability has to decrease under the perturbative probability. Unity, likewise divided by $Z_{p}^{2}$, is plotted in the figure as a full line. Probabilities that do not violate unitarity thus have to remain under this line.

Two calculations with different basis sets are depicted in Fig. 6. The first basis set contains the 22 deepest bound states as well as the states of the positive-energy continuum with energies between $1.1 m c^{2}$ and $3.5 m c^{2}$ and angular momentum quantum numbers $\kappa$ between -2 and +2 . The second basis set in addition contains the analogous states of the negative-energy continuum.

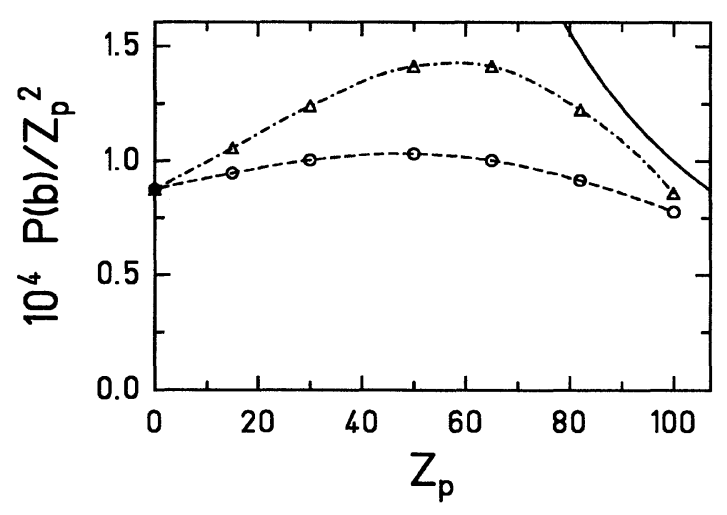

FIG. 6. $K$-shell ionization rate vs $Z_{p}$ for the systems $\mathrm{Pb}^{81+}+Z_{p}$ at the bombarding energy $E=1200 \mathrm{MeV} / \mathrm{u}$ and impact parameter $b=20 \mathrm{fm}$. The probability is divided by $Z_{p}^{2}$, which is the scaling behavior of perturbation theory. $\triangle$ : Without negative-energy continuum; $\bigcirc$ : negative-energy continuum included; full line: unitarity limit. 
Though the excitation of the additional states can be neglected for $t \rightarrow \infty$, they clearly affect the ionization probability. For the considered collision system the ionization rate is reduced by up to $30 \%$ when the negativeenergy states are included.

This effect is caused by the fact that at the distance of closest-approach of the projectile, strong transitions into both continua occur. In particular, the excitation of states of the negative-energy continuum is just one order of magnitude lower than that of states of the positiveenergy continuum. The difference between both continua arises during the outgoing part of the projectile's trajectory. The occupation of the ionization channels does not change considerably after the distance of closest approach, while the occupation probability of the channels of the negative-energy continuum decreases by nearly two orders of magnitude. Obviously the excitation of the ionization channels is disturbed by the negative-energy channels just at that collision time when the strongest excitations occur. For more details cf. Fig. 12(c).

In Fig. 7 the $Z_{p}$-dependent $K$-shell ionization probability is depicted for two different bombarding energies $E=500$ and $E=1200 \mathrm{MeV} / \mathrm{u}$. For higher energies the ionization rate decreases. This is in agreement with calculations by Amundsen and Aashamar [10], who ascertained that for small impact parameters the ionization rate decreases for bombarding energies in the range around $1 \mathrm{GeV} / \mathrm{u}$, while for higher energies the rate becomes constant with corrections of the order $(\ln \gamma) / \gamma^{2}$.

We also briefly discuss $L$-shell ionization. Here we restrict ourselves to the $L_{I}$ subshell, which consists merely of the $2 s$ states. The performed calculations are completely analogous to those for the $K$-shell ionization. We only have to choose the $2 s$ state as the initial state. The corresponding result is also presented in Fig. 7. In contrast to nonrelativistic collisions these calculated data demonstrate that the $L$-shell ionization rate is by a factor of 3 lower than the $K$-shell ionization rate.

Now we want to study the dependence of the ionization rate with respect to the impact parameter. Figure 8

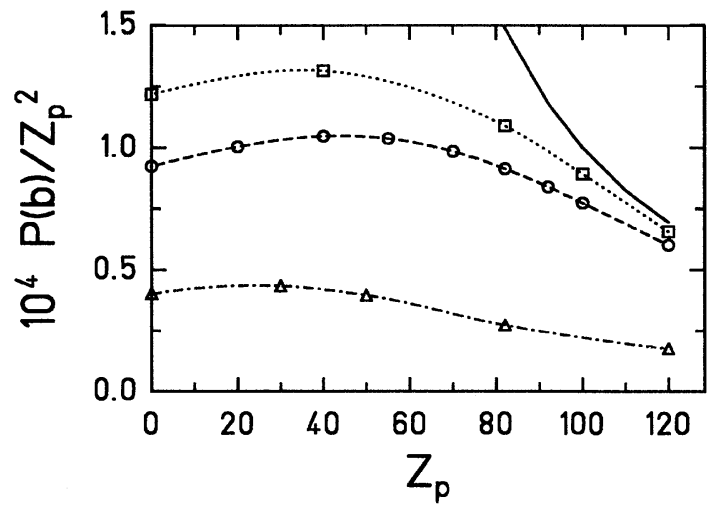

FIG. 7. $K$ - and $L$-shell ionization rate vs $Z_{p}$ for the systems $\mathrm{Pb}^{81+}+Z_{p}$ with impact parameter $b=20 \mathrm{fm}$. The probability is divided by $Z_{p}^{2}$, the scaling behavior of perturbation theory. $\square$ : $K$-shell ionization for $E=500 \mathrm{MeV} / \mathrm{u}$; $\bigcirc: K$-shell ionization for $E=1200 \mathrm{MeV} / \mathrm{u} ; \triangle: L$-shell ionization for $E=1200 \mathrm{MeV} / \mathrm{u}$; full line: unitarity limit.

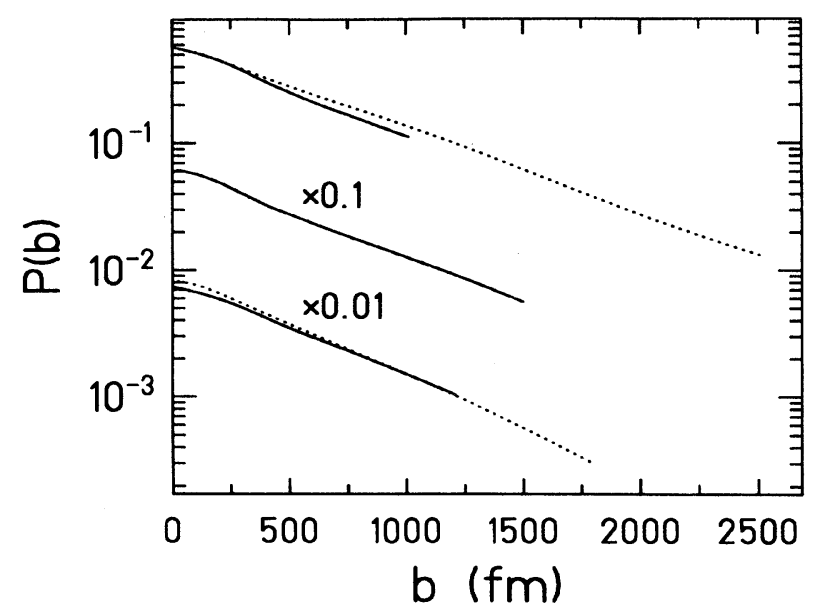

FIG. 8. Dependence of the ionization rate on the impact parameter for the collision system $\mathrm{Pb}-\mathrm{Pb}$ at (from top to bottom) $E=2000, E=1200$, and $E=500 \mathrm{MeV} / \mathrm{u}$. The results for the lower energies are multiplied by 0.1 and 0.01 , respectively. Full lines: coupled-channel calculations; dotted lines: perturbation theory.

shows an exponential decreasing behavior of the ionization rate when the impact parameter is increased. This holds true in perturbation theory as well as for coupledchannel calculations and for all bombarding energies $E=500, E=1200$, and $E=2000 \mathrm{MeV} /$ u.

Figure 9 displays the $\delta$ electron spectra from $\mathrm{Pb}-\mathrm{Pb}$ collisions with $E=2000 \mathrm{MeV} / \mathrm{u}$ with impact parameter $b=10, b=210$, and $b=810 \mathrm{fm}$. As a function of electron energy the spectra decrease exponentially. This decrease is considerably stronger for larger impact parameters.

Obviously the used basis contains the energetically most important channels. The restriction to states with angular momentum quantum numbers $\kappa=-2 \ldots+2$ seems to be more stringent. We presume that the results will be modified by the addition of further angular momenta. Unfortunately, due to computer time and storage reasons it is not possible to enlarge the employed basis considerably.

\section{A. Electron excitation}

The excitation into higher bound states is calculated in the same manner as the ionization; we merely substitute a bound state for the final state. The comparison between ionization and excitation of the $2 s$ as well as of the $3 s$ state is displayed in Fig. 10 for the system $\mathbf{P b}-\mathrm{Pb}$ at 2000 $\mathrm{MeV} / \mathrm{u}$. For large impact parameters the rates decrease, but for the ionization probability the decrease is weaker than for the transitions to bound states. This is readily understood since the used localized wave packets diminish as $1 / r^{2}$, while the bound states exhibit a much stronger exponential decline.

The results of coupled-channel calculations show a weaker decrease for the excitation of bound states than perturbation theory does. This might be explained by multistep processes, in which, e.g., the $2 s$ states is excited 


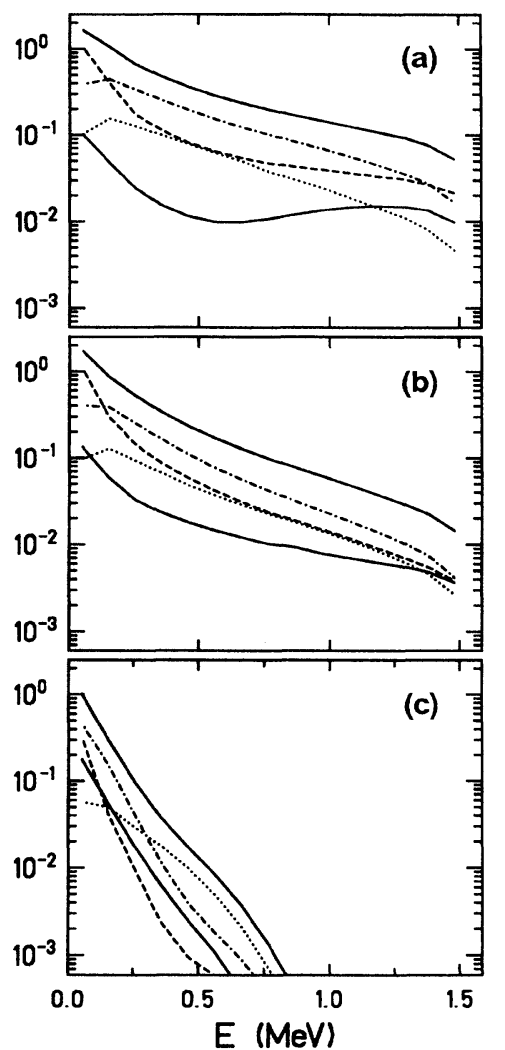

FIG. 9. $\delta$-electron spectrum for the collision system $\mathrm{Pb}-\mathrm{Pb}$ at $E=2000 \mathrm{MeV} / \mathrm{u}$ and impact parameter (a) $b=10 \mathrm{fm}$; (b) $b=210 \mathrm{fm}$; and (c) $b=810 \mathrm{fm}$. The lines display the probability for the excitation into a wave-packet state with the width $\Delta E=0.2 \mathrm{mc}^{2}$. The full curve at the top represents the sum over all angular momenta, while the other curves show the contributions of the different angular momenta. Dashed line: $\kappa=-1$; full line; $\kappa=+1$; dash-dotted line: $\kappa=-2$; dotted line: $\kappa=+2$.

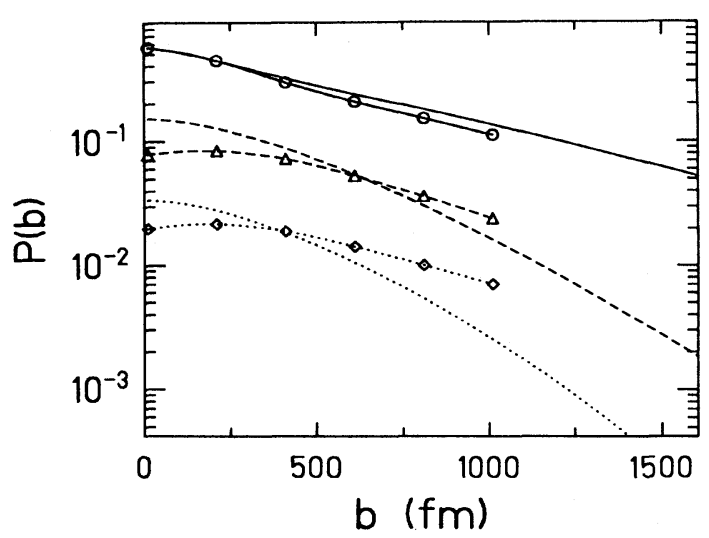

FIG. 10. Excitation of the bound $2 s$ state (dashed line) and $3 s$ state (dotted line), compared with the excitation of continuum states (full line) for the system $\mathrm{Pb}-\mathrm{Pb}$ at $E=2000 \mathrm{MeV} / \mathrm{u}$. The lines marked by circles, triangles, and squares are calculated using the coupled-channel equations, while the other lines represent the result of perturbation theory. indirectly via the continuum states, which are more strongly excited. In fact, for very small impact parameters, we note a slight increase of the probabilities.

Finally, in Fig. 11 we analyze the various spin contributions by depicting the ratio of spin flip to non-spin-flip contributions. For central collisions $(b=0)$ the projectile potential contains only angular momenta with $M=0$. Due to the additive behavior of the magnetic quantum numbers a spin flip is not possible and the ratio diverges. By increasing the impact parameter, the spin flip becomes more and more probable.

\section{B. Pair creation}

In the framework of our formalism, pair creation can be regarded as the excitation of an electron from the negative-energy continuum into a bound state (pair creation with capture) or into a state of the positiveenergy continuum (direct pair creation). Thus the basis that should be used to describe this process has to contain the negative-energy continuum in an appropriate manner.

We make explicit use of the time reversal invariance. As a consequence, a single coupled-channel calculation is sufficient for the calculation of the pair-creation probability with capture of the electron into the $K$ shell of the target.

The dependence of ionization and pair-creation channels on collision time is represented in Fig. 12 assuming different approximations. Figure 12(a) shows the result of perturbation theory, while Figs. 12(b) and 12(c) display results of coupled-channel calculations. In Fig. 12(b) the continuum-continuum interactions are neglected, while in Fig. 12(c) all couplings are included. During the incoming part of the trajectory, differences between the various approximations are hardly visible. Not until $t \approx 0$, when strong excitations occur, do deviations become recognizable. In particular, for the pair creation, relatively large differences are visible during the outgoing

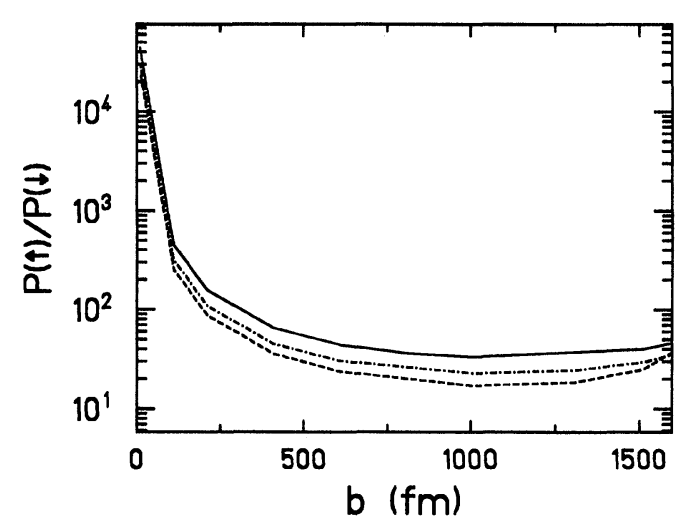

FIG. 11. Ratio of excitations without spin flip and excitations with spin flip $P(\uparrow) / P(\downarrow)$ for the states $2 s$ (dashed) and $3 s$ (dash-dotted) at a bombarding energy $E=2000 \mathrm{MeV} / \mathrm{u}$ in the system $p-\mathrm{Pb}$. Due to the low projectile charge, perturbation theory has been used. The full line represents the same calculation for the $2 s$ states at $E=500 \mathrm{MeV} / \mathrm{u}$. 


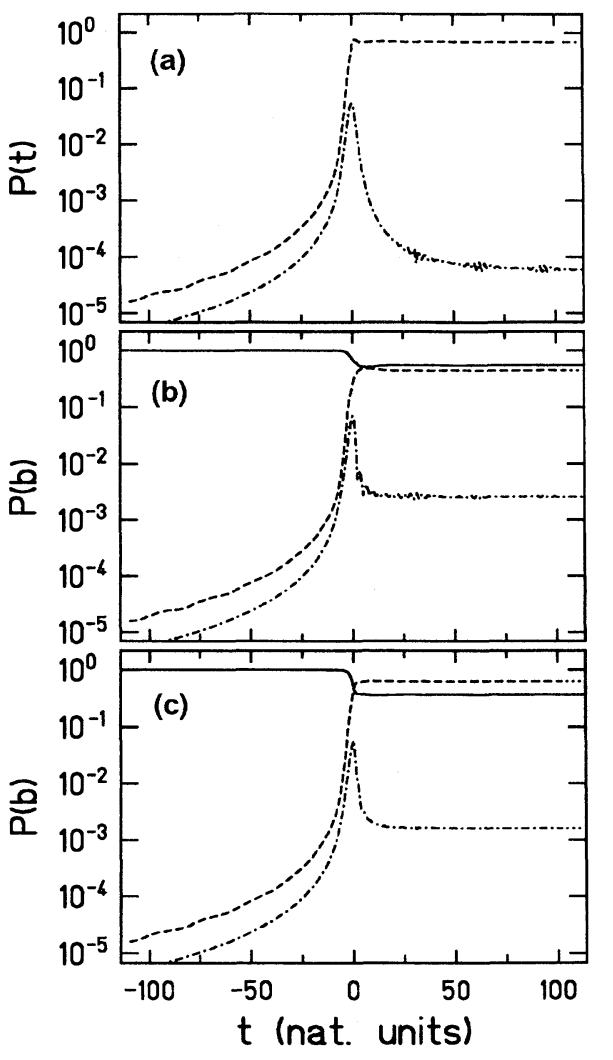

FIG. 12. Excitation of ionization and pair-creation channels during a $\mathrm{Pb}-\mathrm{Pb}$ collision with $E=1200 \mathrm{MeV} / \mathrm{u}$ and $b=20 \mathrm{fm}$. The time $t$ is given in natural units defined by $\hbar=c=m=1$. Full line: $1 s$ state; dashed line: ionization; dash-dotted line: pair creation. (a) Perturbation theory; (b) coupled-channel calculations, continuum-continuum couplings neglected; and (c) coupled-channel calculations, all couplings included.

part of the trajectory. Using perturbation theory, the excitation decreases very much after $t=0$ and finally approaches a low level. However, in the coupled-channel calculations the pair creation decreases much less after the collision and remains more than one order of magnitude above the outcome of perturbation theory.

Comparing both coupled-channel calculations demonstrates that the continuum-continuum couplings are not responsible for this effect. Rather it seems that the approximation $a_{1 s, 1 s} \approx 1$ that forms the basis of perturbation theory is-due to excitation of higher bound states and ionization channels - no longer justified. The depopulation of the $1 s$ state during the collision is responsible for the fact that the probability that flowed into the positron channels cannot flow back completely.

The transition from the validity region of perturbation theory to the nonperturbative behavior $[1,34,35]$ can be deduced by varying the projectile's charge. This is accomplished in Fig. 13. The probabilities are divided by $Z_{p}^{2}$, the scaling behavior in perturbation theory, as in Fig. 6.

Obviously also the pair-creation probability approaches continuously the perturbative value for $Z_{p} \rightarrow 0$.

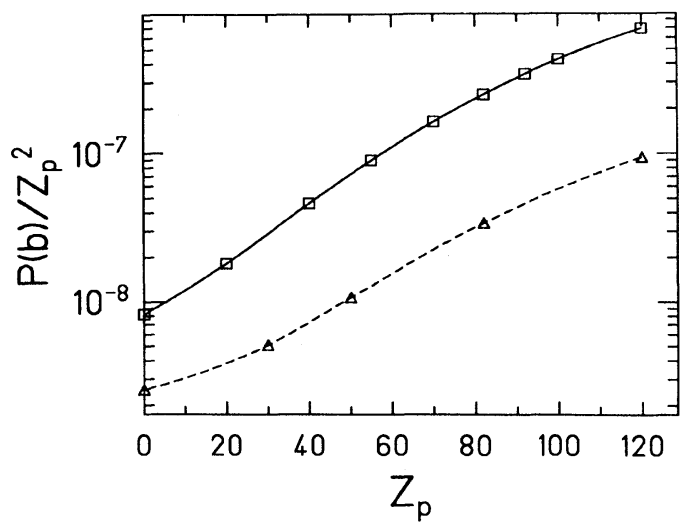

FIG. 13. Pair-creation probability with capture of the electron into the $K$ shell (full line) and in the $L_{I}$ shell (dashed line) of the target as function of the charge of the projectile. The system is $\mathrm{Pb}^{82+}+Z_{p}$ at a bombarding energy $E=1200 \mathrm{MeV} / \mathrm{u}$ and impact parameter $b=20 \mathrm{fm}$. The probability is divided by the scaling behavior of perturbation theory $Z_{p}^{2}$.

But with an increasing nuclear charge of the projectile, the probability for the creation of an electron-positron pair rises very fast over the result of perturbation theory $[1,35]$. At $Z_{p}=92$, i.e., for a totally ionized uranium nucleus as projectile, the result from the coupled-channel calculation is nearly two orders of magnitude above the corresponding result of perturbation theory. Strayer et al. solved the time-dependent Dirac equation on a grid using $B$ splines and likewise found - in the case of muon pair production-large deviations from perturbation theory [34].

For the energies investigated in this paper, the paircreation probabilities are relatively small. This probability increases at higher bombarding energy and finally also violates unitarity. But these high energies cannot be handled by the present coupled-channel calculations employing the basis as indicated above. Nevertheless, Best, Greiner, and Soff [36] showed that the violation of unitarity in these cases does not prohibit the application of perturbative methods. Instead, the sum of the squared amplitudes can be interpreted as the mean number of produced pairs, which of course is not normalized to unity.

Now we want to expand the investigation to negative projectile charges, i.e., to antinuclei. Experimentally, this appears highly unrealistic, since at present it is not possible to produce heavy antinuclei, not to mention a beam of antinuclei. Antiprotons are accessible, but the charge is-as for protons-so small that the difference with perturbation-theory calculations is negligible. However, from a theoretical point of view it is interesting to explore how ionization and pair creation behave for large negative projectile charges in order to point out the influence of strong Coulomb field effects. First results are given in Fig. 14. The ionization probability falls more and more below the result of perturbation theory, while the probability for pair creation decreases only slightly below the perturbative result and then remains constant (remember the scaling with $1 / Z_{p}^{2}$ ). 


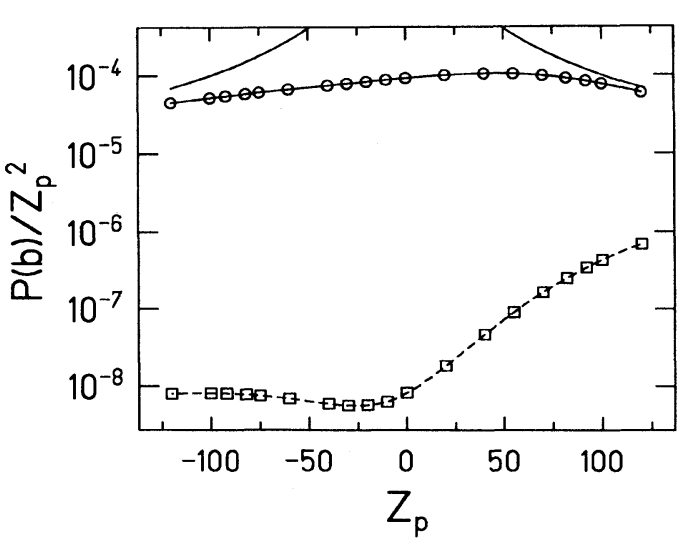

FIG. 14. $K$-shell ionization and pair-creation probability with capture into the $K$ shell of the target as function of the projectile charge $Z_{p}$ for the system $\mathrm{Pb}+Z_{p}$ at the bombarding energy $E=1200 \mathrm{MeV} / \mathrm{u}$ and impact parameter $b=20 \mathrm{fm}$. The probability has been divided by $Z_{p}^{2}$. Dash-dotted line: ionization probability; dashed line: pair-creation probability; full line: unitarity limit.

Finally, in Fig. 15 we display the dependence of pair creation with capture in the $K$ shell on the impact parameter $b$. For small impact parameters the large deviation of perturbation theory from the results of the coupledchannel calculation is recovered. The difference decreases fast with larger impact parameter and practically vanishes at about $600 \mathrm{fm}$. For $b>600 \mathrm{fm}$ it is known from analytical calculations using perturbation theory that the pair-creation rate decreases almost exponentially [37].

For the calculation of pair creation with capture of the electron in the $K$ shell a single coupled-channel calculation was sufficient. The calculation of direct pair creation is more time consuming since every state of the positiveenergy continuum may serve as the final state. We have to perform a full coupled-channel calculation for each basis state of the positive-energy continuum. For reasons of computer time we could not take into account all continuum states. Instead, we restricted ourselves to a few

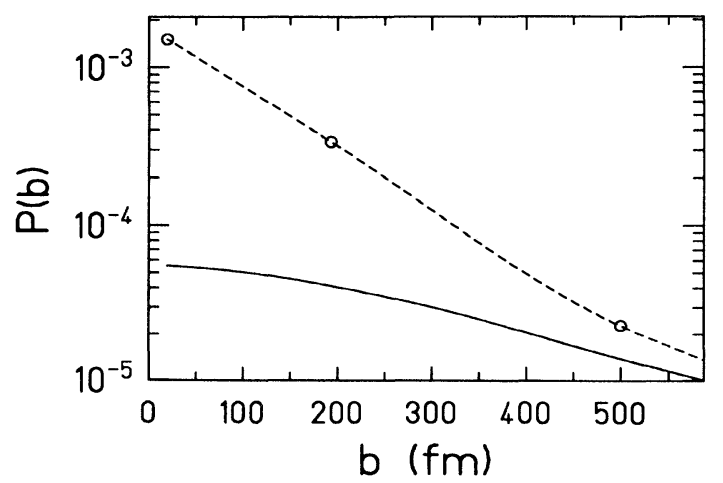

FIG. 15. Pair creation with capture with respect to the impact parameter for the system $\mathrm{Pb}-\mathrm{Pb}, E=1200 \mathrm{MeV} / \mathrm{u}$. Dashed line: coupled-channel calculations; full line: perturbation theory.

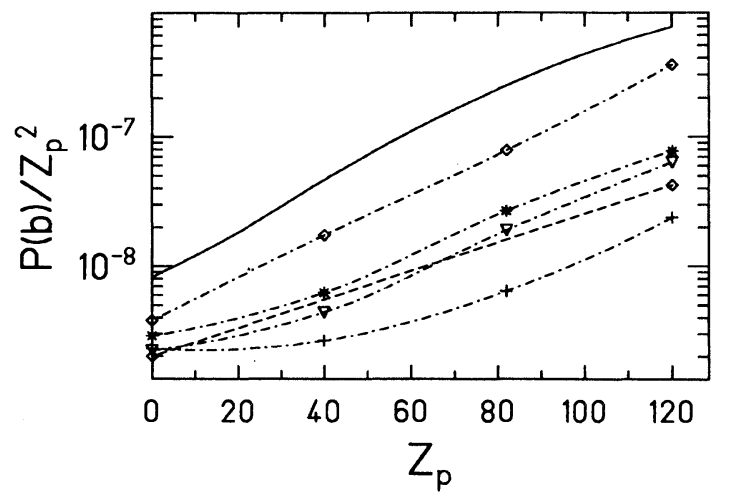

FIG. 16. Direct pair-creation probability vs $Z_{p}$ for the system $\mathrm{Pb}-\mathrm{Pb}$, bombarding energy $E=1200 \mathrm{MeV} / \mathrm{u}$, and impact parameter $b=10 \mathrm{fm}$. Five electron states of the upper continuum are chosen. Full line: Capture into the $K$ shell; dash-dotted line: electron energy $E_{e^{-}}=1.1 \mathrm{mc}^{2}$; dashed line: electron energy $E_{e^{-}}=1.5 \mathrm{mc}^{2} ; \diamond: \kappa=-1 ; \nabla: \kappa=+1 ; \star$ : $^{2} \kappa=-2 ;+$ : $\kappa=+2$.

continuum states with positive energy. Thus, the results correspond to a multiple differential cross section.

In the first place we want to know whether the nonperturbative behavior of pair creation with capture can be found again for direct pair creation. Figure 16 demonstrates that this holds true. The increase over perturbation-theory data is different for different states, but amounts to more than one order of magnitude in all considered cases. This increase is weakest for the highest angular momenta and energies.

\section{COUPLED-CHANNEL EQUATIONS AND GAUGE TRANSFORMATIONS}

The Dirac equation is invariant under local gauge transformations when the minimal coupling is used for the coupling to the electromagnetic field. A local gauge transformation of the complex phase of the wave function causes additional terms in the Dirac equation due to the derivative operators in the Hamiltonian. These additional terms are canceled by the gauge transformation of the electromagnetic potentials. Of course, observables are not changed by this procedure.

Now we want to investigate the influence of a gauge transformation onto the coupled-channel equations. It should be clear that the description of an electron by a complete set of basis states is gauge invariant, since this is an equivalent representation of the Dirac equation. But the question arises whether the results depend on the chosen gauge when using a truncated basis set or even perturbation theory.

Two different requirements for gauge invariance of the coupled-channel equations can be distinguished. The stronger requirement for manifest gauge invariance means that all amplitudes $a_{i}$ at every time step are independent of the gauge. The second requirementwhich is weaker - we call "asymptotic gauge invariance." 
In this case it is merely required that, e.g., in a heavyion collision the coefficients $a_{i}$ are independent of the gauge for $t \rightarrow \pm \infty$, when the perturbing fields vanish. For this it has to be assumed that at the time $t \rightarrow \pm \infty$, when the $a_{i}$ are compared, a fixed gauge is given. But only for vanishing fields a certain gauge is distinguished from all the others, namely $A^{\mu}=0$. This is fulfilled in the case of a heavy-ion collision for $t \rightarrow \pm \infty$. But this condition is not fulfilled in every case, e.g., in an electromagnetic wave of infinite extension (atom in a laser field).

We start with the expansion of a wave function in terms of a complete basis set $\left\{\phi_{k}\right\}$. After a gauge transformation we have

$$
\psi=\sum_{k} a_{k} \phi_{k} \rightarrow \psi^{\prime}
$$

Now there are two possibilities: We may transform $a_{k}$ as well as $\phi_{k}$. If the $a_{k}$ shall remain unchanged we have to write

$$
\phi_{k} \rightarrow \phi_{k}^{\prime}
$$

and since the basis functions $\phi_{k}$ are eigenstates of $H_{0}$,

$$
H_{0} \rightarrow H_{0}^{\prime} \text {. }
$$

Now the coefficients are independent of the chosen gauge and thus can be considered as probability amplitudes without any difficulties concerning their interpretation. We get the manifest gauge invariant formulation of the coupled-channel equations when all quantities are written from the beginning in a gauge invariant manner $[38,39]$. In this case it results that $H_{0}$ has to contain the spatial components of the electromagnetic potential. Thus the manifest gauge invariance leads to a complicated form of $H_{0}$ and a complicated calculation of the basis wave functions. Different aspects and examples of gauge transformations from this point of view are discussed in a series of publications by Kobe and co-workers [40-43].

But the manifest gauge invariance is not necessary and $H_{0}$ can be chosen in such a way that the basis states are easy to calculate and constant in time. Then the coefficients $a_{k}$ in Eq. (54) have to be transformed. In the following we want to investigate whether in this case the asymptotic gauge invariance remains fulfilled [44].

\section{A. Perturbation theory}

The coupled-channel equations read

$$
\dot{a}_{f}=-i \sum_{k} a_{k}\left\langle\phi_{f}|\hat{V}| \phi_{k}\right\rangle e^{i \Delta E_{f k} t}
$$

with the electromagnetic potentials $\hat{V}=e \hat{\boldsymbol{\alpha}} \cdot \mathbf{A}-e V$ and the energy difference $\Delta E_{f k}=E_{f}-E_{k}$. Inserting the gauge transformed electromagnetic potentials

$$
\hat{\boldsymbol{V}}^{\prime}=\hat{\boldsymbol{V}}-e \hat{\boldsymbol{\alpha}} \cdot \nabla \chi-e \partial_{t} \chi,
$$

we get

$$
\begin{aligned}
\dot{a}_{f}^{\prime}= & -i \sum_{k} a_{k}^{\prime}\left\langle\phi_{f}|\hat{\boldsymbol{V}}| \phi_{k}\right\rangle e^{i \Delta E_{f k} t} \\
& +i \sum_{k} a_{k}^{\prime}\left\langle\phi_{f}\left|e \hat{\boldsymbol{\alpha}} \cdot \nabla \chi+e \partial_{t} \chi\right| \phi_{k}\right\rangle e^{i \Delta E_{f k} t} .
\end{aligned}
$$

In order to simplify this expression, we first consider the matrix element

$$
\begin{aligned}
\left\langle\phi_{f}|\hat{\boldsymbol{\alpha}} \cdot \boldsymbol{\nabla} \chi| \phi_{k}\right\rangle & =\int d V \phi_{f}^{\dagger} \hat{\boldsymbol{\alpha}} \phi_{k} \cdot \nabla \chi \\
& =-\int d V \boldsymbol{\nabla}\left(\phi_{f}^{\dagger} \hat{\boldsymbol{\alpha}} \phi_{k}\right) \chi+\int_{\partial V} d \boldsymbol{\sigma} \phi_{f}^{\dagger} \hat{\boldsymbol{\alpha}} \phi_{k} \chi .
\end{aligned}
$$

In the following we require

$$
\chi(r \rightarrow \infty)=\chi(t \rightarrow \pm \infty)=0 .
$$

This boundary condition causes a vanishing surface integral in (60). Using the continuity equation of the transition currents of the stationary basis wave functions $\phi_{k}$,

$$
i \Delta E_{f k} \phi_{f}^{\dagger} \phi_{k}+\nabla\left(\phi_{f}^{\dagger} \hat{\alpha} \phi_{k}\right)=0,
$$

we proceed

$$
\left\langle\phi_{f}|\hat{\boldsymbol{\alpha}} \cdot \nabla \chi| \phi_{k}\right\rangle=i \Delta E_{f k} \int d \boldsymbol{V} \phi_{f}^{\dagger} \phi_{k} \chi \equiv i \Delta E_{f k}\left\langle\phi_{f}|\chi| \phi_{k}\right\rangle \text {. }
$$

Thus it follows

$$
\left\langle\phi_{f}\left|\hat{\boldsymbol{\alpha}} \cdot \nabla \chi^{\dagger} \partial_{t} \chi\right| \phi_{k}\right\rangle e^{i \Delta E_{f k} t}=\partial_{t}\left\langle\phi_{f}\left|\chi e^{i \Delta E_{f k} t}\right| \phi_{k}\right\rangle \text {. }
$$

This relation is inserted in (59) and results in

$$
\begin{aligned}
\dot{a}_{f}^{\prime}= & -i \sum_{k} a_{k}^{\prime}\left\langle\phi_{f}|\hat{V}| \phi_{k}\right\rangle e^{i \Delta E_{f k} t} \\
& +i e \sum_{k} a_{k}^{\prime} \partial_{t}\left\langle\phi_{f}\left|\chi e^{i \Delta E_{f k}{ }^{t}}\right| \phi_{k}\right\rangle .
\end{aligned}
$$

Since the additional term from the gauge transformation is a total time derivative, we derive the result that in first-order perturbation theory

$$
a_{f}^{\prime}(t=\infty)=a_{f}(t=\infty) \text { for } \chi(t= \pm \infty)=0
$$

is valid, i.e., the asymptotic gauge invariance is fulfilled.

We already mentioned that for a complete set of basis states the coupled-channel equations are asymptotically gauge independent. Rumrich, Soff, and Greiner [44] proved this explicitly in the representation of the coupled channels. In addition, an example has been given for the gauge dependence when using a truncated basis.

\section{B. Gauge transformation of the target potential}

Now we want to discuss a slightly different point of view. Instead of transforming the projectile potential according to Eq. (58),

$$
\hat{V}^{\prime}=\hat{V}-\Delta \hat{V}=\hat{V}-e \hat{\alpha} \cdot \nabla \chi-e \partial_{t} \chi,
$$

likewise we may transform the target potential that is contained in $\hat{H}_{0}$ :

$$
\hat{H}_{0}^{\prime}=\hat{H}_{0}-\Delta \hat{V} \text {. }
$$

If at the same time the basis wave functions are changed according to

$$
\phi^{\prime}=\phi \exp (i \chi) \text {, }
$$

the Dirac equation for the basis states remains unchanged: 


$$
i \partial_{t} \phi^{\prime}=\hat{H}_{0}^{\prime} \phi^{\prime} \text {. }
$$

Thus the only effect for the coupled-channel equations (7) is a substitution of the basis wave functions by the transformed wave functions $\phi^{\prime}$. But $\phi^{\prime}$ differs from $\phi$ only by a phase factor that is the same for all basis states and thus cancels in the matrix elements:

$$
\left\langle\phi_{f}^{\prime}|\hat{V}| \phi_{k}^{\prime}\right\rangle=\left\langle\phi_{f}|\hat{V}| \phi_{k}\right\rangle \text {. }
$$

Therefore a gauge transformation of the target potential does not influence the coupled-channel equations at all and thus may be ignored. But in principle the initial conditions have to be changed according to

$$
\begin{aligned}
\psi_{i}(t \rightarrow-\infty) & =\phi_{i}(t \rightarrow-\infty) \\
& =\sum_{k} a_{k i}(t \rightarrow-\infty) \phi_{k}^{\prime}(t \rightarrow-\infty),
\end{aligned}
$$

which in general is possible only in a complete basis set. But requiring the initial conditions (61) for the gauge field, we have $\phi_{i}(t \rightarrow-\infty)=\phi_{i}^{\prime}(t \rightarrow-\infty)$ and also the initial conditions remain unchanged.

Now we turn to an alternative description of (projectile) gauge transformations and show the connection to target gauge transformations and to changes of the basis set. We split the total Hamiltonian $\hat{H}=\hat{H}_{0}+\widehat{V}$ in a different way:

$$
\hat{H}=\hat{H}_{0}^{\prime}+\hat{V}^{\prime},
$$

where

$$
\begin{aligned}
& \hat{H}_{0}^{\prime}=\hat{H}_{0}+\Delta \hat{V}, \\
& \hat{V}^{\prime}=\hat{V}-\Delta \hat{V}, \\
& \Delta \hat{V}=e \hat{\alpha} \cdot \nabla \chi+e \partial_{t} \chi .
\end{aligned}
$$

The transition from $\hat{H}_{0}$ to $\hat{H}_{0}^{\prime}$ is just a target gauge transformation and thus may be omitted without changing the equations. What remains is a gauge transformation of the projectile potential, which we already considered in detail.

Thus a projectile gauge transformation may be considered as the change of the basis by a (space and time dependent) phase factor

$$
\phi_{k}^{\prime}=\phi_{k} \exp (-i \chi) \text {. }
$$

This again elucidates the fact that only for a complete basis set the coupled-channel equations are gauge invariant. Only in the case of a complete basis set does the transition to another basis not change anything at all.

\section{Coulomb boundary conditions}

Toshima and Eichler [45] demonstrated that the basis wave functions for the coupled-channel equations can be modified in such a way that the resulting interactions between these basis states become short-range interactions. The necessary modifications of the wave functions [45]

$$
\phi^{\infty}(\mathbf{r}, t)=\phi(\mathbf{r}, t) \exp (i \chi)
$$

with

$$
\chi=\frac{e^{2} Z_{P}}{v_{P}} \ln \left(v_{P} t^{\prime}+R^{\prime}\right)
$$

and

$$
R^{\prime}=\left[b^{2}+\gamma^{2}\left(v_{P} t-h z\right)^{2}\right]^{1 / 2}
$$

obviously is just described by a gauge transformation as also pointed out in [6].

Toshima and Eichler [45] solved the coupled-channel equations, taking into account the Coulomb boundary conditions. Their result clearly shows a reduced interaction range compared with calculations not taking into account these boundary conditions. With first-order perturbation theory the result for $t \rightarrow \infty$ remains unchanged. This is in agreement with our more general examination of the influence of gauge transformation on perturbation theory.

In addition to the reduction of the interaction range the result of the coupled-channel calculation displays large differences of the occupation probabilities for $t \rightarrow \infty$. This is due to the incompleteness of the used basis, since in a complete basis the result is independent of the basis.

\section{SUMMARY AND CONCLUSION}

In this paper we presented coupled-channel calculations for the nonperturbative description of relativistic heavy-ion collisions. The time-dependent Dirac equation has been solved by expanding the wave function into the atomic basis set and the resulting system of ordinary coupled differential equations is solved numerically. The angular integrals of the required matrix elements have to be calculated by numerical quadrature. For the matrix elements one should exploit the symmetry relations derived in Sec. II.

The most important result deduced from the coupledchannel calculations is that the probability for the creation of an electron-positron pair during the collision is strongly underestimated by first-order perturbation theory. At a first glance this is amazing since the probability is small compared with unity. The breakdown of perturbation theory is caused by the depopulation of the initial state by ionization and excitation.

Concerning gauge transformations we demonstrated that results obtained from perturbation theory remain unchanged under gauge transformations. However, the solution of the coupled-channel equations is gauge invariant only in the case of a complete basis set. Since the use of Coulomb boundary conditions represents a specific gauge, differences compared with calculations that do not include these boundary conditions [45] are caused by the incompleteness of the employed basis. 
[1] K. Rumrich, K. Momberger, G. Soff, W. Greiner, N. Grün, and W. Scheid, Phys. Rev. Lett. 66, 2613 (1991).

[2] U. Becker, N. Grün, K. Momberger, and W. Scheid, Atomic Processes in Relativistic Heavy Ion Collisions, Vol. 153 of NATO Advanced Study Institute, Series B: Physics, edited by W. Greiner (Plenum, New York, 1987), p. 609.

[3] K. Momberger, N. Grün, W. Scheid, and U. Becker, J. Phys. B 23, 2293 (1990).

[4] N. Toshima and J. Eichler, Phys. Rev. A 38, 2305 (1988).

[5] N. Toshima and J. Eichler, Phys. Rev. A 40, 125 (1989).

[6] J. Eichler, Phys. Rep. 193, 167 (1990).

[7] R. Anholt, S. Nagamiya, J. O. Rasmussen, H. Bowman, J. G. Ioannou-Yannou, and E. Rauscher, Phys. Rev. A 14, 2103 (1976).

[8] D. M. Davidović, B. L. Moiseiwitsch, and P. H. Norrington, J. Phys. B 11, 847 (1978).

[9] R. Anholt, Phys. Rev. A 19, 1004 (1979).

[10] P. A. Amundsen, and K. Aashamar, J. Phys. B 14, 4047 (1981).

[11] S. R. Valluri, U. Becker, N. Grün, and W. Scheid, J. Phys. B 17, 4359 (1984).

[12] R. Anholt, Phys. Rev. A 31, 3579 (1985).

[13] H. Gould, Lawrence Berkeley Laboratory Report No. LBL-18593, 1984 (unpublished).

[14] R. Anholt, W. E. Meyerhof, Ch. Stoller, E. Morenzoni, S. A. Andriamonje, J. D. Molitoris, O. K. Baker, D. H. H. Hoffmann, H. Bowman, J.-S. Xu, Z.-Z. Xu, K. Frankel, D. Murphy, K. Crowe, J. O. Rasmussen, Phys. Rev. A 30, 2234 (1984).

[15] R. Anholt, W. E. Meyerhof, H. Gould, Ch. Munger, J. Alonso, P. Thieberger, and H. E. Wegner, Phys. Rev. A 32, 3302 (1985).

[16] R. Anholt, W. E. Meyerhof, X.-Y. Xu, H. Gould, B. Feinberg, R. J. McDonald, H. E. Wegner, and P. Thieberger, Phys. Rev. A 36, 1586 (1987).

[17] W. E. Meyerhof, R. Anholt, J. Eichler, H. Gould, Ch. Munger, J. Alonso, P. Thieberger, and H. E. Wegner, Phys. Rev. A 32, 3291 (1985).

[18] S. Kelbch, J. Ullrich, W. Rauch, H. Schmidt-Böcking, M. Horbatsch, R. M. Dreizler, S. Hagmann, R. Anholt, A. S. Schlachter, A. Müller, P. Richard, Ch. Stoller, C. L. Cocke, R. Mann, W. E. Meyerhof, and J. D. Rasmussen, J. Phys. B 19, L47 (1986).

[19] H. Berg, R. Dörner, C. Kelbch, S. Kelbch, J. Ullrich, S. Hagmann, P. Richard, H. Schmidt-Böcking, A. S. Schlachter, M. Prior, H. J. Crawford, J. M. Engelage, I.
Flores, D. H. Loyd, J. Pedersen, and R. E. Olson, J. Phys. B 21, 3929 (1988).

[20] J. Bang and J. M. Hansteen, Mat. Fys. Medd. Dan. Vid. Selsk. 31, 13 (1959).

[21] R. K. Smith, B. Müller, and W. Greiner, J. Phys. B 8, 75 (1975).

[22] J. S. Briggs, Rep. Prog. Phys. 39, 217 (1976).

[23] U. Becker, N. Grün, W. Scheid, and G. Soff, Phys. Rev. Lett. 56, 2016 (1986).

[24] J. Thiel, A. Bunker, K. Momberger, N. Grün, and W. Scheid, Phys. Rev. A 46, 2607 (1992).

[25] R. Matzdorf, G. Soff, and G. Mehler, Z. Phys. D 6, 5 (1987).

[26] R. Matzdorf, B. Fricke, and G. Soff, Z. Phys. D 17, 233 (1990).

[27] J. D. Jackson, Classical Electrodynamics (Wiley, New York, 1983).

[28] H.-J. Bär and G. Soff, Physica 128C, 225 (1985).

[29] G. Mehler, G. Soff, K. Rumrich, and W. Greiner, Z. Phys. D 13, 193 (1989).

[30] L. F. Shampine and M. K. Gordon, Computer Solutions of Ordinary Differential Equations: The Initial Value Problem (Freeman, San Francisco, 1975).

[31] J. F. Reading, Phys. Rev. A 8, 3262 (1973).

[32] J. Reinhardt, B. Müller, W. Greiner, and G. Soff, Phys. Rev. Lett. 43, 1307 (1979).

[33] G. Soff, J. Reinhardt, B. Müller, and W. Greiner, Z. Phys. A 294, 137 (1980).

[34] M. R. Strayer, C. Bottcher, V. E. Oberacker, and A. S. Umar, Phys. Rev. A 41, 1399 (1990).

[35] K. Momberger, N. Grün, and W. Scheid, Z. Phys. D 18, 133 (1991).

[36] Ch. Best, W. Greiner, and G. Soff, Phys. Rev. A 46, 261 (1992).

[37] K. Momberger (unpublished).

[38] D. H. Kobe and A. L. Smirl, Am. J. Phys. 46, 624 (1978).

[39] K.-H. Yang, Ann. Phys. 101, 62 (1976).

[40] D. H. Kobe, E. C.-T. Wen, and K.-H. Yang, Phys. Rev. D 26, 1927 (1982).

[41] D. H. Kobe and P. K. Kennedy, J. Phys. B 16, L443 (1983).

[42] D. H. Kobe and K.-H. Yang, Am. J. Phys. 51, 163 (1983).

[43] D. H. Kobe and S. M. Golshan, J. Phys. A 20, 2813 (1987).

[44] K. Rumrich, G. Soff, and W. Greiner, Phys. Lett. A 149, 17 (1990).

[45] N. Toshima and J. Eichler, Phys. Rev. A 42, 3896 (1990). 